\title{
On the impactfulness of theory and review articles
}

\author{
Robert A. Peterson • Victoria L. Crittenden
}

Received: 24 April 2012 / Accepted: 24 April 2012 /Published online: 9 May 2012

(C) Academy of Marketing Science 2012

\begin{abstract}
Every article in the AMS Review should have an impact on marketing thought. The editors of the journal offer several operational guidelines for crafting theoretical/ conceptual manuscripts and suggest three criteria for assessing future impactfulness of a contribution. The marketing professoriate may require a new mindset in order to contribute high quality, impactful theory and review articles that can keep pace with marketplace events and marketing practice.
\end{abstract}

In an ideal world, every article published in the AMS Review will have a substantial impact on marketing thought. Indeed, the editors of the Review, and most likely the authors of articles in the Review, hope that, in some fashion, the articles appearing in the Review - whether theoretical contributions, review contributions, or conceptual clarifications-will add significantly to what is known about marketing.

Greatly oversimplifying, theoretical articles can contribute to marketing knowledge by explaining phenomena and existing conceptual relationships between phenomena and by positing and predicting conceptual relationships to be investigated in the future. As such, a major role of theory is guiding and stimulating empirical research. Review articles (and especially those based on meta-analyses) can contribute to marketing knowledge by integrating existing empirical research findings

\section{R. A. Peterson}

Office of the Vice President for Research,

The University of Texas at Austin,

Austin, TX 78713, USA

e-mail: rap@mail.utexas.edu

V. L. Crittenden $(\triangle)$

Carroll School of Management, Boston College,

Chestnut Hill, MA 02467, USA

e-mail: victoria.crittenden@bc.edu and bringing closure to a particular research area through conclusory statements. Consequently, in an abstract sense, the contributions of theoretical articles and review articles are complementary.

The goal of publishing impactful articles in the $A M S$ Review is simultaneously laudable and lofty, and its achievement benefits the Review, journal authors, and the readership of the journal. The Review benefits through an increase in stature and ranking relative to "competitive journals," thereby attracting more submissions that lead to more impactful articles. Authors of impactful articles similarly benefit through enhanced prestige that presumably leads to higher salaries, rewards, recognition, and so forth. The readership benefits by increasing its knowledge base and creative capacity.

By their very nature, theoretical articles and review articles tend to be more impactful than empiricallygrounded articles, methodological articles, or modelingbased articles. This generalization is supported by Yadav (2010) and illustrated by the ten "best-of-the-best" highimpact articles published in the Review's sister journal, the Journal of the Academy of Marketing Science as of the end of 2011 (Hult and Ferrell 2012) and Cropanzano's (2009) analysis of the most-cited articles in the Journal of Management. However, the generalization must be tempered by the meaning of the term "impactful." What differentiates a very impactful theoretical or review article from one that is not so impactful, and how does one go about creating an impactful theory or review article? Moreover, what does it mean to be "impactful" in the context of academic journals generally or marketing knowledge in particular?

From time-to-time, editors in various academic disciplines have opined on the characteristics of impactful articles. In many instances, the characteristics of impactful articles have been addressed from what might be termed a 
"reverse perspective." Rather than focusing on the characteristics of impactful articles, editors have tended to report the characteristics of manuscripts that were submitted to a journal for publication consideration but rejected. This is probably because it is very difficult to predict whether a given manuscript will become an impactful article (this "result" occurs in the marketplace of ideas but may not occur until an editorial term is over) and editors most likely focus on the characteristics of rejected manuscripts in attempts to improve the quality of manuscripts submitted to their journal. (See, for example, Beyer et al. 1995; Kilduff 2006; Ortinau 2010; Rindova 2008; Stewart 2002; Varadarajan 1996, 2003; Whetten 1989). Although there are exceptions, in general, a rejected manuscript will not or cannot become an impactful article. One widely cited exception that comes to mind is "The Market for Lemons." George Akerlof won the Nobel Prize for this theoretical article, which was ultimately published in the Quarterly Review of Economics after being rejected by The American Economic Review, The Review of Economic Studies, and the Journal of Political Economy.

\section{Crafting impactful theory and review articles}

As one might expect, there is a plethora of articles, books, and papers on how to research, position, and write impactful theory or review articles (e.g., Bem 1995; Cropanzano 2009; Short 2009). Assuming that an underlying theory being constructed possesses substance or that the topic of a review merits dissemination, perhaps the first and foremost operational guideline for achieving an impactful article is learning, understanding, and appreciating the fact that, as Hunt (2011) has advised repeatedly, developing an impactful theory or review article requires creatively crafting a compelling "story" (see also Bem 1995; Rindova 2008; Summers 2001). The term "crafting" is intentionally used to emphasize the notions of "art," "special skill," "attention to detail," and so forth that are necessary to construct a manuscript that may ultimately become an impactful article. The term "story" is likewise intentionally used to indicate that a theory or review manuscript seeking to become a theory or review article must have a clear and coherent beginning, middle, and end (conclusion).

A second operational guideline to increase the probability of a manuscript becoming an impactful article is to obtain input from colleagues on the manuscript prior to submitting it to a journal for publication consideration. Colleagues should be asked to perform a pseudo-review of the manuscript. In particular, they should be asked to evaluate the manuscript and answer questions such as, "Why would this manuscript be rejected for publication?" or "What is missing from this proposed theory or review?" Merely soliciting general comments from colleagues, such as "What do you think about this manuscript?", almost invariably results in praise or no actionable suggestions that would improve the manuscript. When asking for input, oftentimes good insights can be obtained from colleagues who are experts in the area of the research as well as from those who are not. The latter can point out communication problems that authors or experts might overlook because they have "too much" knowledge of the research area.

In addition to these operational guidelines (that actually can be applied to any manuscript preparation process), there are certain characteristics of theory and review articles that increase the likelihood of their being impactful. Three such characteristics are importance of the topic, "interestingness," and timing. It goes without saying that if a topic is judged to be unimportant, any theory or review article focusing on it is not likely to generate much traction or even attention. In brief, the foundation of any impactful theory or review article is a unique (important) "Big Idea" that sets the article apart from other competing articles seeking to be impactful and which is, in and of itself, a significant contribution to marketing knowledge.

The more interesting a theory or review article is, that is, the more surprising - and even counter-intuitive - the results or conclusions are, the greater the likelihood of it becoming impactful. This characteristic was observed by Davis (1971) in a frequently referenced article appropriately titled "That's Interesting" and has been the subject of discussion in articles attempting to explain factors influencing article impactfulness (e.g., Short 2009; Smith 2003). It is also often mentioned in passing in articles addressing measures of impactfulness (e.g., Sternberg \& Gordeeva 1996; Stewart 2002).

A third characteristic determining the impactfulness of a theory or review article is timing. Serendipity influences impactfulness. As Mount and Barrick (1998) acknowledge, their highly impactful article was "the right article at the right time" (p. 854). Likewise, Sternberg and Gordeeva's (1996) investigation revealed the importance of an article appearing "at the right moment" (p.75) in determining its impactfulness. Thus, to a certain extent, whether an article becomes impactful is a function of timing... and luck.

\section{Assessing impactfulness}

There are two general ways to measure the impactfulness of a theory or review article. One way is typically termed subjective or qualitative, whereas the other is termed objective or quantitative. The impactfulness of a theory or review article can be assessed subjectively by recording the number of times the article has been reprinted in textbooks, edited volumes or collected works. Similarly, the extent to which it is used in university courses, whether it has been mentioned in the popular press, or if it has been the subject of a special 
session in a formal seminar or conference can be used to assess subjective impactfulness. Moreover, subjective impactfulness can be assessed by surveying researchers to determine their absolute and relative perceptions of the article. Although subjective assessment provides a holistic perspective of impactfulness, by definition it is difficult to carry out and communicate a subjective assessment in any structured fashion.

Objective or quantitative assessment of the impactfulness of a theory or review article has traditionally been done by means of a citation analysis (e.g., Bettencourt \& Houston 2001). A citation analysis can simply report the number of citations an article has obtained or report some calculated value based on the number of citations, such as the impact factor, immediacy index, and citation half-life (e.g., Amin \& Mabe 2000; Glanzel \& Moed 2002).

While citation analyses are becoming increasingly incorporated in journal, individual, and departmental evaluations of "productivity," "relevance," and "quality" (e.g., Durden \& Ellis 1993), caution is needed so that what is being assessed is correctly interpreted and to ensure that any one citation analysis is meaningful (e.g., Hecht et al. 1998). Because article citation rates differ across citation cultures, academic disciplines, and journal policies, it is often very difficult to compare counts or indices.

Moreover, truly impactful articles have a tendency to not have citation rates commensurate with their impact. Consider Cronbach's (1951) seminal article, "Coefficient Alpha and the Internal Structure of Tests." This article changed the conceptual and measurement paradigm for test and scale reliability. Although the article has been cited more than 16,000 times in Google Scholar, the number of Google citations underestimates its applications. This is because coefficient alpha has become an icon, and, analogous to other icons, it is often no longer associated with its creator or its originating article. Stated somewhat differently, its attribution or citation history in publications has evolved from the 1951 Psychometrika article to "Cronbach's coefficient alpha" to "coefficient alpha," to "alpha," with no specific article citations. Indeed, there is a tendency to incorrectly cite the source of Cronbach's coefficient alpha by attributing it to a textbook.

In brief, analogous to beauty- "I know it when I see it" - assessing the impactfulness of a theory or review article is fraught with ambiguity, inconsistency, and subjectivity. Even so, article impactfulness is a worthy goal and should remain one.

\section{Take away}

For the AMS Review to be credible and attract outstanding manuscripts that may become impactful articles, it is imperative that the Review nurture and mentor both high potential authors and manuscripts. This requires new mindsets for authors, reviewers, editors, doctoral students, professors, and even marketing departments as well as, perhaps, new journal procedures for attracting and processing manuscripts. The marketing discipline needs high quality, impactful theory and review articles if it is to keep pace with marketplace events and marketing practice.

Thirty years ago Thompson (1981) wrote a brief editorial in which he summarized succinctly the characteristics of an impactful article. Paraphrasing Thompson, impactful articles convey "a message which is important, timely, and relevant...interesting and well-written" and possess "originality of one type or another" (p. 1). Thompson's conclusions are as valid today for theory and review articles as they were three decades ago. Nearly two decades later, Mount and Barrick (1998, p. 856) wrote that "writing a highly cited [impactful] article is a low probability event that involves hard work, creativity, good timing, and luck." This conclusion also remains valid today and reflects a wisdom that should be shared.

\section{References}

Amin, M., \& Mabe, M. (2000). Impact factors: Use and abuse. Perspectives in Publishing, 1, 1-6.

Bem, D. J. (1995). Writing a review article for Psychological Bulletin. Psychological Bulletin, 118, 172-177.

Bettencourt, L. A., \& Houston, M. B. (2001). The impact of article method type and subject area on article citations and reference diversity in $J M, J M R$, and JCR. Marketing Letters, 12, 327-340.

Beyer, J. M., Chanove, R. G., \& Fox, W. B. (1995). The review process and the fates of manuscripts submitted to AMJ. Academy of Management Journal, 38, 1219-1260.

Cronbach, L. J. (1951). Coefficient alpha and the internal structure of tests. Psychometrika, 16, 297-334.

Cropanzano, R. (2009). Writing nonempirical articles for Journal of Management: General thoughts and suggestions. Journal of Management, 35, 1304-1311.

Davis, M. S. (1971). That's interesting! Towards a phenomenology of sociology and a sociology of phenomenology. Philosophy of the Social Sciences, 1, 309-344.

Durden, G. C., \& Ellis, L. V. (1993). A method for identifying the most influential articles in an academic discipline. Atlantic Economic Journal, 21, 1-10.

Glanzel, W., \& Moed, H. F. (2002). Journal impact measures in bibliometric research. Scientometrics, 53, 171-193.

Hecht, F., Hecht, B. K., \& Sandberg, A. A. (1998). The journal "impact factor": A misnamed, misleading, misused measure. Cancer Genetics and Cytogenetics, 104, 77-81.

Hult, G. T. M., \& Ferrell, O. C. (2012). A tribute to forty years of toplevel marketing research. Journal of the Academy of Marketing Science, 40, 1-7.

Hunt, S. D. (2011). Developing successful theories in marketing: Insights from resource-advantage theory. AMS Review, 1, 72-84.

Kilduff, M. (2006). Editor's comments: Publishing Theory. Academy of Management Review, 31, 252-255. 
Mount, M. K., \& Barrick. (1998). Five reasons why the "Big Five" article has been frequently cited. Personnel Psychology, 51, 849-857.

Ortinau, D. J. (2010). Primer for new authors: On the fundamentals of publishing in marketing journals. Journal of Marketing Theory and Practice, 18, 91-100.

Rindova, V. (2008). Editor's comments: Publishing theory when you are new to the game. Academy of Management Review, 33, 300-303.

Short, J. (2009). The art of writing a review article. Journal of Management, 35, 1312-1317.

Smith, D. C. (2003). The importance and challenges of being interesting. Journal of the Academy of Marketing Science, 31, 319-322.

Sternberg, R. J., \& Gordeeva, T. (1996). The anatomy of impact: What makes an article influential? Psychological Science, 7, 69-75.

Stewart, D. W. (2002). Getting published: Reflections of an old editor. Journal of Marketing, 66, 1-6.
Summers, J. O. (2001). Guidelines for conducting research and publishing in marketing: From conceptualization through the review process. Journal of the Academy of Marketing Science, 29, 405415.

Thompson, J. H. (1981). Essential qualities of a good manuscript. Journal of Small Business Management, 19, 1-2.

Varadarajan, P. R. (1996). From the editor: Reflections on research and publishing. Journal of Marketing, 60, 3-6.

Varadarajan, P. R. (2003). Musings on relevance and rigor of scholarly research in marketing. Journal of the Academy of Marketing Science, 31, 368-376.

Whetten, D. A. (1989). What constitutes a theoretical contribution? Academy of Management Review, 14, 490-495.

Yadav, M. S. (2010). The decline of conceptual articles and implications for knowledge development. Journal of Marketing, 74, 1-19. 